\title{
The changing landscape of infective endocarditis in South Africa
}

\author{
M C de Villiers, ${ }^{1} \mathrm{MB}$ ChB; C A Viljoen, ${ }^{2} \mathrm{MB} \mathrm{ChB}, \mathrm{MMed}, \mathrm{FCP}(\mathrm{SA}) ; \mathrm{K}$ Manning, ${ }^{1} \mathrm{BSc}, \mathrm{PG}$ Dip (Diet), MSc (Med); \\ C van der Westhuizen, ${ }^{1} \mathrm{MB} \mathrm{ChB} ; \mathrm{A}$ Seedat, ${ }^{1} \mathrm{MB} \mathrm{ChB} ; \mathrm{M}$ Rath, ${ }^{1} \mathrm{MB} \mathrm{ChB} ; \mathrm{M}$ Graham, ${ }^{1} \mathrm{MB} \mathrm{ChB} ; \mathbf{M}$ Ntsekhe, ${ }^{2} \mathrm{MD}, \mathrm{PhD}, \mathrm{FACC}$ \\ ${ }^{1}$ Department of Medicine, Faculty of Health Sciences, University of Cape Town and Groote Schuur Hospital, Cape Town, South Africa \\ ${ }^{2}$ Division of Cardiology, Department of Medicine, Faculty of Health Sciences, University of Cape Town and Groote Schuur Hospital, \\ Cape Town, South Africa
}

Corresponding author: M Ntsekhe(mpiko.ntsekhe@uct.ac.za)

\begin{abstract}
Background. Little is known about the current clinical profile and outcomes of patients with infective endocarditis (IE) in South Africa (SA). Objectives. To provide a contemporary and descriptive overview of IE in a representative SA tertiary centre.

Methods. We conducted a retrospective review of the records of patients admitted to Groote Schuur Hospital, Cape Town, between 2009 and 2016 fulfilling universal criteria for definite or possible IE, in search of demographic, clinical, microbiological, echocardiographic, treatment and outcome information.

Results. A total of 105 patients fulfilled the modified Duke criteria for IE. The median age of the cohort was 39 years (interquartile range (IQR) 29 - 51), with a male preponderance (61.9\%). The majority of the patients (72.4\%) had left-sided native valve endocarditis, $14.3 \%$ had right-sided disease, and $13.3 \%$ had prosthetic valve endocarditis. A third of the cohort had rheumatic heart disease. Although $41.1 \%$ of patients with left-sided disease had negative blood cultures, the three most common organisms cultured in this subgroup were Staphylococcus aureus (18.9\%), Streptococcus spp. (16.7\%) and Enterococcus spp. (6.7\%). Participants with right-sided endocarditis were younger (29 years, IQR 27 - 37) and were mainly intravenous drug users (73.3\%), and the majority cultured positive for S. aureus (73.3\%) with frequent septic pulmonary complications (40.0\%). The overall in-hospital mortality was $16.2 \%$, with no deaths in the group with rightsided endocarditis. Predictors of death in our patients were heart failure (odds ratio (OR) 8.16, 95\% confidence interval (CI) 1.77 - 37.70 ; $p=0.007)$ and age $>45$ years (OR $4.73,95 \%$ CI $1.11-20.14 ; p=0.036)$. Valve surgery was associated with a reduction in mortality (OR 0.09 , 95\% CI $0.02-0.43 ; p=0.001)$.

Conclusions. IE remains an important clinical problem in a typical teaching tertiary care centre in SA. In this setting, it continues to affect mainly young people with post-inflammatory valve disease and congenital heart disease. The in-hospital mortality associated with IE remains high. Intravenous drug-associated endocarditis caused by S. aureus is an important IE subset, comprising $\sim 10 \%$ of all cases, which was not reported 15 years ago, and culture-negative endocarditis remains highly prevalent. Heart failure in IE carries a significant risk of death and needs a more intensive level of care in hospital. Finally, cardiac surgery was associated with reduced mortality, with the largest impact in patients with heart failure.
\end{abstract}

S Afr Med J 2019;109(8):592-596. DOI:10.7196/SAMJ.2019.v109i8.13888

Despite advances in diagnostic imaging, antimicrobial treatment and cardiac surgery, infective endocarditis (IE), as defined by the modified Duke criteria, ${ }^{[1]}$ remains a potentially fatal condition with an annual in-hospital mortality rate of $\sim 20 \% .^{[2,3]}$ Observational data from the developed world indicate that the profile of IE has changed significantly over the past four decades. IE has become a disease that predominantly affects older and/or diabetic patients with infections on degenerative native valves or prosthetic valves. Rheumatic heart disease (RHD) has all but disappeared as a predisposing risk factor. ${ }^{[4-6]}$ The classic clinical signs of IE are now infrequent, patients often presenting with only fever and/ or a heart murmur. ${ }^{[4]}$ Furthermore, endocarditis caused by oral organisms (such as Streptococcus viridans) and culture-negative endocarditis have both declined significantly as a proportion of IE in the developed world. ${ }^{[4]}$ Nosocomial infections from longterm indwelling catheters and intravenous lines are increasing in incidence, and Staphylococcus aureus, coagulase-negative S. aureus and Enterococcus spp. are the dominant organisms. ${ }^{[7]}$ In spite of this evolution in the patient and clinical profile of IE, in-hospital mortality, morbidity and complication rates remain high. Specifically, congestive cardiac failure, stroke and intracardiac abscesses with conduction abnormalities are reported at frequencies of $\sim 60 \%, 30 \%$ and $1-15 \%$, respectively. ${ }^{[8-11]}$

Apart from a recent focus on intravenous (IV) drug use (IVDU)related $\mathrm{IE}^{\left[{ }^{[12]}\right.}$ there are few contemporary data on IE from South Africa (SA) or sub-Saharan Africa (SSA), with the most recent information on the subject published in $2003 .{ }^{[13]}$ Specifically, there is a dearth of information on patient profiles, risk factors, predisposing cardiac conditions, and the spectrum of causative microbiological organisms. Furthermore, little is known about the prevalence and predictors of complications, the proportion of patients with IE who undergo surgery, and in-hospital mortality rates in SSA. This knowledge is vital for increasing the survival of IE patients as well as improving their overall care by optimising local clinical practice.

\section{Objectives}

This study was conducted to address these gaps in our local knowledge, providing a contemporary and descriptive overview of IE in a representative SA tertiary centre. 


\section{Methods}

This was a retrospective review of patient records. Patients aged $>17$ years with complete data who fulfilled the modified Duke criteria for $\mathrm{IE}^{[14]}$ and were commenced on treatment for possible or definite IE at Groote Schuur Hospital in Cape Town in 2009 - 2016 were included. After approval by the University of Cape Town's Human Research Ethics Committee (ref. no. 517/2016), 181 potential cases were identified by screening the hospital's electronic database for all hospital admissions and referrals to cardiology. Demographic, clinical, microbiological, echocardiographic, laboratory and outcome data were collected on all identified cases.

Six independent investigators (MCdV, CAV, CvdW, AS, MR and MG) used a standardised data collection form, from which data were entered into REDCap (Research Electronic Data Capture, a secure online database manager hosted at the University of Cape Town). All the collected data were verified. Collected data were exported from the REDCap database to Stata software, version 14.1 (StataCorp, USA), for statistical analysis. Descriptive statistics were used to summarise demographic, clinical and microbiological variables. Univariable and multivariable logistic regression analysis was used to determine the association between clinically relevant variables and inpatient mortality in patients with left-sided IE. Variables were selected a priori and were excluded if data were sparse. Those considered for regression analysis were age, sex, comorbidities (including HIV status), serum creatinine, white cell count, organism cultured, echocardiographic complications, clinical complications and valve replacement surgery performed. Variables were retained in the multivariable model if the $p$-value was $<0.25$ or if an association had been demonstrated in previous studies. ${ }^{[15,16]}$ Unadjusted and adjusted odds ratios (ORs) were presented with $95 \%$ confidence intervals (CIs).

\section{Results}

One hundred and five cases were included in the study (Fig. 1), with 64.8\% ( $n=68)$ and $35.8 \%(n=37)$ of patients fulfilling the modified Duke criteria for definite and possible IE, respectively. In all cases, the responsible clinicians felt it appropriate to commence treatment for endocarditis. There was a steady increase in the number of cases seen over the period from 2009 to 2016, with a peak in 2014 (Supplementary File 1, available at http://www.samj.org.za/public/ sup/Supplementary.pdf).

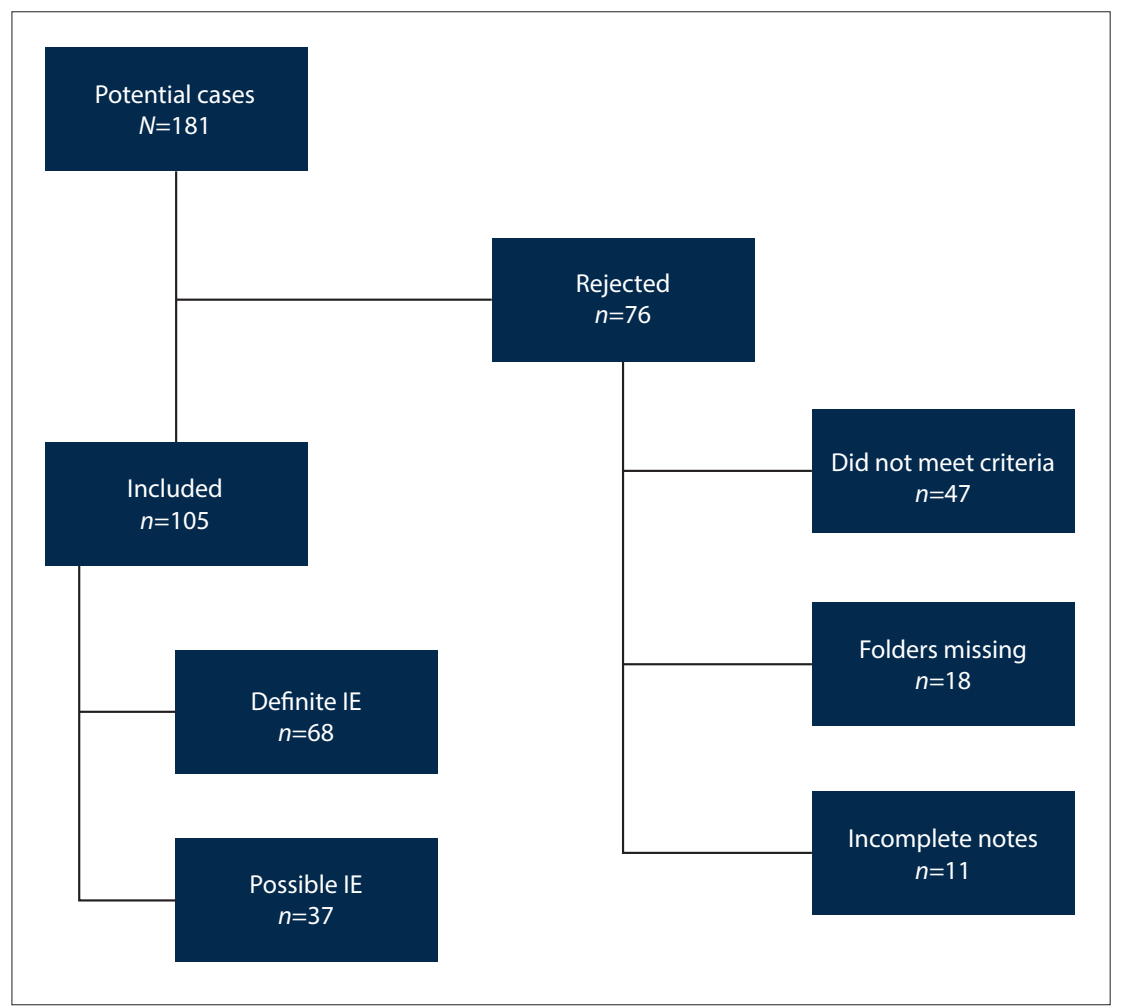

Fig. 1. Patients included in the study. (IE = infective endocarditis.)

The median age of the cohort was 39 years (interquartile range (IQR) 29 - 51), with a slight male preponderance $(61.9 \%)$. The majority of the patients had left-sided native valve endocarditis (72.4\%), while the remainder were evenly split between prosthetic valve endocarditis (13.3\%) and right-sided endocarditis (14.3\%).

The most common underlying cardiac conditions were RHD (34.3\%), mechanical or tissue prosthetic valves (13.3\%; most of these patients had had valve surgery for RHD), and congenital heart disease (10.5\%). Five patients $(4.8 \%)$ had a prior history of IE and 1 patient had device-associated endocarditis (permanent pacemaker). Among those who were tested for HIV (87/105 patients), $23.0 \%$ were seropositive. Ten patients $(9.5 \%)$ had diabetes mellitus, while other comorbidities such as chronic kidney disease, chronic liver disease and cancer were uncommon (3 patients each). One patient had a permanent IV port. Table 1 gives a detailed description of the clinical characteristics, special investigations and laboratory results. The most frequent presenting signs and symptoms were fever (63.8\%), dyspnoea (61.0\%), a temperature $>38.5^{\circ} \mathrm{C}(58.1 \%)$ and weight loss (24.8\%). Most patients (85.7\%) had a new or changed murmur on clinical examination. More than a quarter (27.6\%) had an acute neurological deficit, and $18.1 \%$ were noted to have splinter haemorrhages (Table 2).
All patients had a transthoracic echocardiogram. Where vegetations were detected, the mitral valve $(52.9 \%)$ and aortic valve (37.6\%) were most frequently affected. Two-thirds $(68.4 \%)$ of the patients with aortic valve involvement had severe aortic regurgitation, whereas $54.1 \%$ of those with mitral involvement and $36.4 \%$ of those with tricuspid involvement had severe regurgitation.

The median number of blood cultures done per patient was 3 (IQR 2.5 - 4.5). Blood cultures were persistently positive after 48 hours in $21.0 \%$ of patients in this study (13.2\% of left-sided native endocarditis, $42.9 \%$ of prosthetic valve endocarditis and $40.0 \%$ of right-sided endocarditis; $p=0.006$ ). Based on the attending clinician's evaluation, the most frequent sources of infection were thought to be the skin (66.7\%), oral cavity $(25.9 \%)$ and gastrointestinal tract $(7.9 \%)$. Of patients with left-sided disease, $40.7 \%$ had negative blood cultures (Fig. 2, A), and the three most common organisms cultured in this subgroup were S. aureus (18.7\%), Streptococcus spp. (16.5\% (S. viridans $9.9 \%$, other 6.6\%)) and Enterococcus spp. (6.6\%).

Right-sided disease differed from leftsided disease. Patients with tricuspid and pulmonary valve endocarditis were younger than those with left-sided disease (median age 29.1 years, IQR 27 - 37) and had a greater male predominance (73.3\%), IVDU was a common risk behaviour $(73.3 \%)$ and 
Table 1. Demographics, comorbidities, clinical characteristics and mortality of patients with left- and right-sided IE

\begin{tabular}{|c|c|c|c|}
\hline & Total $(N=105)$ & Left-sided disease $(N=90)$ & Right-sided disease $(N=15)$ \\
\hline Sex male, $n(\%)$ & $65(61.9)$ & $54(60.0)$ & $11(73.3)$ \\
\hline Age (years), median (IQR) & $39(29-51)$ & $42.7(31-53)$ & $29.1(27-37)$ \\
\hline Congenital heart disease, $n(\%)$ & $11(10.5)$ & $9(10.0)$ & $2(13.3)$ \\
\hline RHD, $n(\%)$ & $36(34.3)$ & $36(40.0)$ & 0 \\
\hline Previous IE, $n(\%)$ & $5(4.8)$ & $5(5.6)$ & 0 \\
\hline Prosthetic valves, $n(\%)$ & $14(13.3)$ & $14(15.6)$ & 0 \\
\hline No comorbidities, $n(\%)$ & $31(29.5)$ & $20(22.2)$ & $11(73.3)$ \\
\hline HIV, $n(\%)$ & $20 / 87(23.0)$ & $20 / 75(26.6)$ & $0 / 12$ \\
\hline IVDU, $n(\%)$ & $15(14.2)$ & $4(4.5)$ & $11(73.3)$ \\
\hline Diabetes mellitus, $n(\%)$ & $10(9.5)$ & $9(10.0)$ & $1(6.7)$ \\
\hline Ethanol use, $n(\%)$ & $23(21.9)$ & $23(21.9)$ & 0 \\
\hline Native valve IE, $n(\%)$ & $91(86.7)$ & $76(84.4)$ & $15(100)$ \\
\hline Prosthetic valve IE, $n(\%)$ & $14(13.3)$ & $14(15.6)$ & 0 \\
\hline Duke criteria definite, $n(\%)$ & $68(64.8)$ & $53(58.9)$ & $15(100)$ \\
\hline \multicolumn{4}{|l|}{ Echocardiography } \\
\hline LVEF, \% (IQR) & $60(53-66)$ & $59.5(53-65)$ & $64(58-70.5)$ \\
\hline Vegetations present, $n(\%)$ & $85(81.0)$ & $71(78.9)$ & $14(93.3)$ \\
\hline Aortic regurgitation, $n(\%)$ & $39(37.1)$ & $38(42.2)$ & $1(6.7)$ \\
\hline Mitral regurgitation, $n(\%)$ & $62(59.1)$ & $60(66.7)$ & $2(13.3)$ \\
\hline Pulmonary regurgitation, $n(\%)$ & $9(8.6)$ & $8(8.9)$ & $1(6.7)$ \\
\hline Tricuspid regurgitation, $n(\%)$ & $34(32.4)$ & $23(21.9)$ & $11(73.3)$ \\
\hline \multicolumn{4}{|l|}{ Laboratory values } \\
\hline WCC $\left(\times 10^{9} / \mathrm{L}\right)$, median $(\mathrm{IQR})$ & $12.7(8.3-15.7)$ & $11.5(7.7-15.4)$ & $14.95(13.8-18.5)$ \\
\hline $\mathrm{Hb}(\mathrm{g} / \mathrm{dL})$, median $(\mathrm{IQR})$ & $10.1(8.5-11.4)$ & $10.3(8.8-11.4)$ & $9.15(7-11.6)$ \\
\hline Creatinine $(\mu \mathrm{mol} / \mathrm{L})$, median (IQR) & $89.5(67-120)$ & $87(67-110)$ & $121.5(62-209)$ \\
\hline Diuretic use, $n(\%)$ & $62 / 100(59.1)$ & $58 / 86(64.5)$ & $4 / 14(28.6)$ \\
\hline Valvular surgery performed, $n(\%)$ & $44(42.3)$ & $43(47.8)$ & $1(6.7)$ \\
\hline Inpatient mortality, $n(\%)$ & $17(16.2)$ & $17(18.9)$ & 0 \\
\hline
\end{tabular}

Table 2. Presenting signs and symptoms of patients with left- and right-sided IE

\begin{tabular}{llll}
\hline & Total $(\boldsymbol{N}=\mathbf{1 0 5}), \boldsymbol{n}(\%)$ & Left-sided disease $(\boldsymbol{N}=\mathbf{9 0}), \boldsymbol{n}(\%)$ & Right-sided disease $(\boldsymbol{N}=\mathbf{1 5}), \boldsymbol{n}(\%)$ \\
\hline Fever & $67(63.8)$ & $54(60.0)$ & $13(86.7)$ \\
Dyspnoea & $64(61.0)$ & $57(61.5)$ & $7(46.7)$ \\
Poor appetite & $13(12.4)$ & $12(13.3)$ & $1(6.7)$ \\
Weight loss & $26(24.8)$ & $22(24.4)$ & $4(26.7)$ \\
Neurological deficit & $29(27.6)$ & $28(31.1)$ & $1(6.7)$ \\
Arthralgia & $11(10.5)$ & $8(8.9)$ & $3(20.0)$ \\
Temperature $>38.5^{\circ} \mathrm{C}$ & $61(58.1)$ & $51(56.7)$ & $10(66.7)$ \\
Clubbing & $32(30.5)$ & $28(31.1)$ & $4(26.7)$ \\
New or changed murmur & $90(85.7)$ & $79(87.8)$ & $11(73.3)$ \\
Splinter haemorrhages & $19(18.1)$ & $17(18.9)$ & $2(13.3)$ \\
Haematuria on urine test strip & $44 / 64(68.8)^{*}$ & $35 / 54(64.8)$ & $9 / 10(90.0)$ \\
IE $=$ infective endocarditis. & & & \\
${ }^{*}$ Data missing on 41 patients. & & &
\end{tabular}

S. aureus was the most common organism cultured (73.3\%), while $13.3 \%$ of patients were culture-negative (Fig. 2, B).

\section{In-hospital course and outcome}

Heart failure was the most common in-hospital complication (42.9\%) (Table 3), followed by embolic complications (36.1\%), acute kidney injury (21.9\%) and pneumonia (8.6\%). In-hospital mortality was observed only in patients with left-sided native and prosthetic valve endocarditis, with a rate of $18.9 \%$ in that subgroup. Heart failure (adjusted OR 8.16, 95\% CI 1.77 - 37.70; $p=0.007$ ) and age
$>45$ years (adjusted OR 4.73, 95\% CI $1.11-20.14 ; p=0.036$ ) were significantly associated with an increased in-hospital mortality in this study (Table 4); however, data sparsity reduced the precision of these estimates. HIV, diabetes and infection with $S$. aureus did not appear to influence mortality. The most common complications for right-sided disease were septic pulmonary emboli (40.0\%) and acute kidney injury (40.0\%).

All patients were treated with antibiotics, and 44 (42.3\%) underwent valve surgery. Heart failure was the most common indication for surgery $(66.7 \% ; p=0.001)$. Surgery was associated with a reduced risk 
of death (OR 0.09, 95\% CI $0.02-0.43$; $p=0.001$ ). Only one patient with right-sided endocarditis underwent surgery, i.e. tricuspid valve repair due to severe tricuspid regurgitation.

\section{Discussion}

We have presented a retrospective review of the hospital records of patients admitted to an SA urban tertiary referral centre with a diagnosis of IE. The study, which covered the period between 2009 and 2016, is the first of its kind since the early 2000s and describes the demographic, clinical and microbiological profile of patients with the disease, discusses treatment strategies, and provides information about in-hospital outcomes and their predictors.

The main findings of this study demonstrate that IE in urban SA remains a disease of relatively young people (median age 39 years) with rheumatic or congenital heart disease (44.8\%); culture-negative endocarditis remains a prevalent problem; the proportion of IE caused by community-acquired S. aureus appears to be growing; and there is evidence that IVDU-associated rightsided endocarditis is now an important problem. Furthermore,

\begin{tabular}{ll} 
Table 3. Complications in patients with IE $(\boldsymbol{N}=\mathbf{1 0 5})$ \\
\hline & $\boldsymbol{n}(\mathbf{\%})$ \\
\hline Heart failure & $45(42.9)$ \\
Embolic & $38(36.1)$ \\
Ischaemic stroke & $15(14.3)$ \\
Mycotic aneurysm & $8(7.6)$ \\
Septic pulmonary emboli & $6(5.7)$ \\
Pulmonary infarct & $2(1.9)$ \\
Splenic infarct & $2(1.9)$ \\
Cerebral abscess & $2(1.9)$ \\
Intracranial haemorrhage & $3(2.9)$ \\
Acute kidney injury & $23(21.9)$ \\
Pneumonia & $9(8.6)$ \\
Septic shock & $7(6.7)$ \\
IE = infective endocarditis. &
\end{tabular}

we found that heart failure was the most common complication of IE, was the most important predictor of death and was the main reason patients were referred for cardiac surgery, an intervention that was associated with reduced mortality rates. Finally, HIV, diabetes, alcohol use and chronic kidney disease were not important predictors of a poor outcome.

Although no major conclusions can be made from a retrospective review, or from comparisons with other studies, it is interesting to compare our findings with data from recent international registries ${ }^{[4,17-20]}$ and a similar but older SA study. ${ }^{[13]}$ When compared with our findings, these registries suggest that there are major differences in the age profile ( $>70$ years $\mathrm{v}$. $<40$ years), clinical profile (degenerative valve disease v. RHD) and microbiological profile of the studied IE patients, with apparently fewer cases of culturenegative and oral organism-related IE seen in the developed world.

Additionally, in comparison with findings from the earlier SA study, ${ }^{[13]}$ the main observed differences revolved around the microbiological profile, proportion of IVDU-associated right-sided disease, and prevalence of underlying congenital heart disease.

Whereas community-associated S. aureus was noted infrequently in 2003 , it was found in $26 \%$ of our overall cohort and was the dominant cause of infection in patients with IVDU-associated IE. This is in line with a recently published study from Johannesburg, which found S. aureus to be present in $61 \%$ of recreational drug users who developed IE. ${ }^{[12]}$ The same study reported a higher burden of right-sided endocarditis at their centre compared with our findings. This finding could possibly be explained by recent data on drug abuse in SA that showed higher rates of IVDU in Gauteng Province than in the Western Cape. ${ }^{[21]}$ On the other hand, viridans group streptococci, which were previously dominant, may be diminishing in frequency. The high rate of culture-negative IE in our cohort was in line with the previous study and other low- and middle-income country (LMIC) data, as demonstrated in a systematic review by Njuguna et al. ${ }^{[22]}$ High rates of empirical administration of antibiotics prior to blood culture collection are often cited as the main reason for high culture-negative IE rates. Of patients with culture-negative IE in our study, $24.8 \%$ had received antibiotics prior to blood culture collection. Culture-
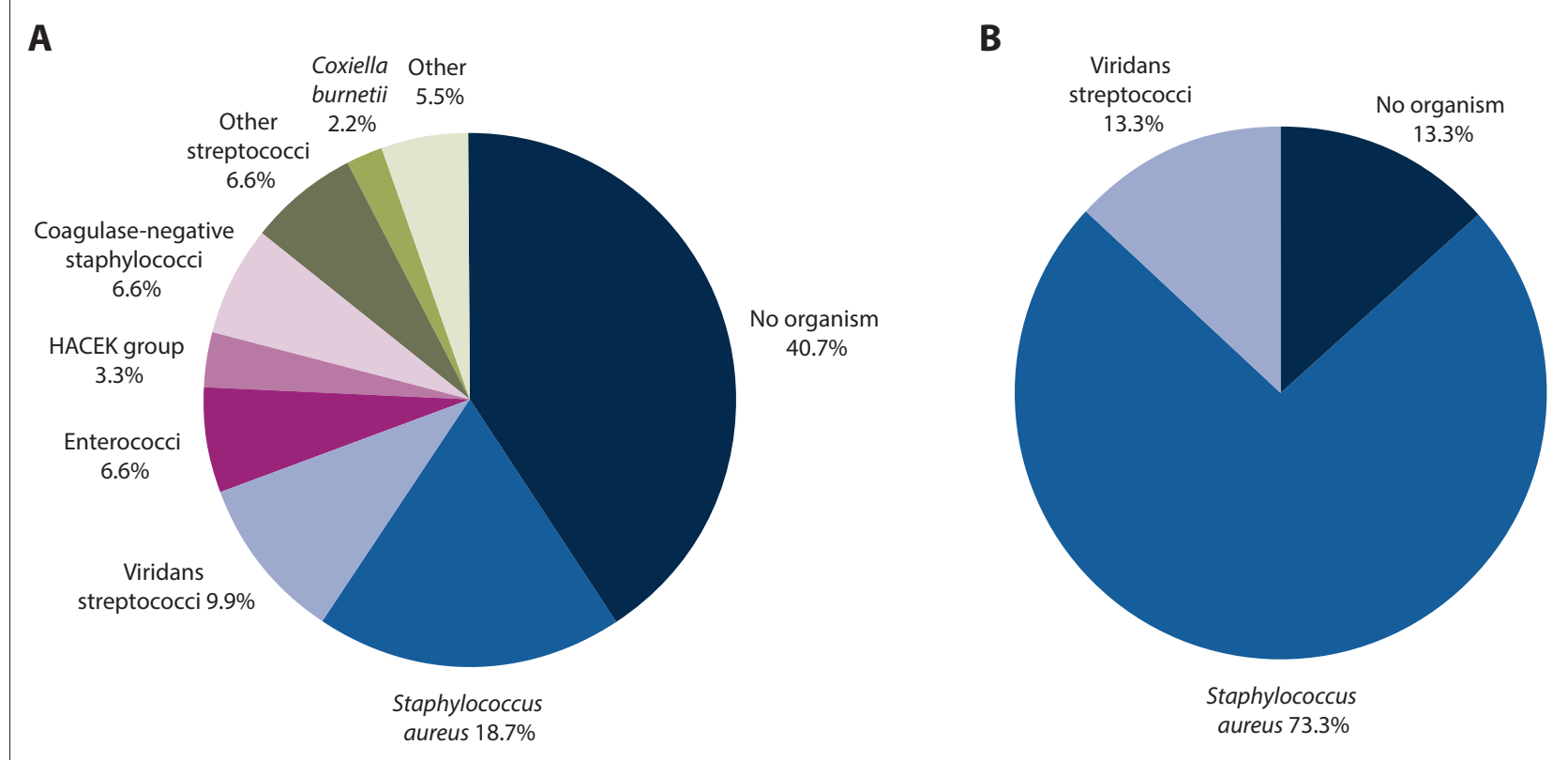

Fig. 2. Microbiological profile of (A) left-sided and (B) right-sided IE. (IE = infective endocarditis; HACEK = Haemophilus spp., Aggregatibacter spp., Cardiobacterium hominis, Eikenella corrodens and Kingella spp., a group of fastidious Gram-negative bacteria that are an unusual cause of IE.) 
Table 4. Univariable and multivariable logistic regression analysis of clinically relevant risk factors associated with inpatient mortality in patients with left-sided endocarditis*

\begin{tabular}{|c|c|c|c|c|}
\hline & $\begin{array}{l}\text { Univariable regression analysis, } \\
\text { unadjusted OR }(95 \% \mathrm{CI})\end{array}$ & $p$-value & $\begin{array}{l}\text { Multivariable regression analysis, } \\
\text { adjusted OR }(95 \% \mathrm{CI})\end{array}$ & $p$-value \\
\hline Age $>45$ years & $3.73(1.26-11.09)$ & 0.018 & $4.73(1.11-20.14)$ & 0.036 \\
\hline Diabetes mellitus & $2.39(0.53-10.73)$ & 0.225 & $0.85(0.12-6.15)$ & 0.870 \\
\hline Staphylococcus aureus & $1.42(0.40-5.06)$ & 0.589 & $2.04(0.41-10.23)$ & 0.386 \\
\hline Heart failure & $1.83(0.63-5.34)$ & 0.269 & $8.16(1.77-37.70)$ & 0.007 \\
\hline Valvular surgery performed & $0.18(0.05-0.67)$ & 0.011 & $0.09(0.02-0.43)$ & 0.001 \\
\hline
\end{tabular}

$\mathrm{OR}=$ odds ratio; $\mathrm{CI}=$ confidence interval; $\mathrm{IE}=$ infective endocarditis

${ }^{*}$ Analysis was restricted to patients with left-sided IE, as risk profiles differ between right- and left-sided IE. There were insufficient data to conduct a similar analysis in the right-sided cohort.

negative rates are significantly lower in higher-income countries $(10 \%) \cdot{ }^{[4]}$

Mortality in our cohort (16.2\%) was lower than figures previously reported in other LMICs $(19-46 \%) .{ }^{[22]}$ A possible explanation for this relatively lower than expected mortality was the liberal use of cardiac surgery for patients with international guideline-based recommendations such as heart failure. ${ }^{[23]}$ Approximately $48 \%$ of patients with left-sided IE underwent surgery, a much higher figure than those reported in other LMIC registries, where $\sim 15 \%$ receive valve replacement for the same indication. ${ }^{[22,24]}$

\section{Study strengths and limitations}

To the best of our knowledge, this is the largest cohort of IE patients in SSA to date and provides important information and insight into the disease in an SA context. There are a number of important limitations of this study, including that it was a single-centre, retrospective study; that the number of participants was relatively small; that the subgroup sizes were relatively small and unequal; that it is likely that not all patients with IE over the given period of interest were captured; and that some patient records may have been incomplete and their International Statistical Classification of Diseases and Related Health Problems, 9th revision (ICD-9) and ICD-10 information may not always have been accurate. As a result of these and other limitations inherent in the design and conduct of such a study, caution needs to be exercised with regard to the conclusions drawn as well as the generalisability of the findings.

\section{Conclusions}

IE in a typical teaching tertiary care centre in SA remains an important clinical problem. In this setting, it continues to affect mainly young people with post-inflammatory valve disease and congenital disease and is associated with in-hospital mortality that is quite high at almost $20 \%$. Intravenous drug-associated endocarditis caused by S. aureus is an important IE subset, comprising $\sim 10 \%$ of all cases, which was not reported 15 years ago, and culture-negative endocarditis remains highly prevalent. Heart failure in IE carries a significant risk of death and needs a more intensive level of care in hospital. Finally, cardiac surgery reduced mortality and had most impact in patients with heart failure. These findings suggest that prospective cohorts of IE are needed to keep track of changing trends and profiles in order to provide clinicians with more robust information to inform their clinical practice and thereby improve patient outcomes.

Declaration. This publication forms part of MCdV's MMed degree in Internal Medicine, University of Cape Town.

Acknowledgements. We thank Nicholas Simpson for his excellent assistance, and the Records Department of Groote Schuur Hospital for assisting with acquiring patient folders to collect data.
Author contributions. MCdV developed the study protocol, collected and analysed data and wrote the manuscript. CAV and MN contributed to the study design, data analysis and reviewing and editing of the manuscript. KM contributed to data analysis. CvdW, CAV, AS, MR and MG contributed to data collection.

Funding. None.

Conflicts of interest. None.

1. Li JS, Sexton DJ, Mick N, et al. Proposed modifications to the Duke criteria for the diagnosis of infective endocarditis. Clin Infect Dis 2000;30(4):633-638. https://doi.org/10.1086/313753

Thuny F, Grisoli D, Collart F, Habib G, Raoult D. Management of infective endocarditis: Challenges Thuny F, Grisoli D, Collart F, Habib G, Raoult D. Management of infective endocarditis: Challenges
and perspectives. Lancet 2012;379(9819):965-975. https://doi.org/10.1016/s0140-6736(11)60755-1

and perspectives. Lancet 2012;379(9819):965-975. https://doi.org/10.1016/s0140-6736(11)60755-1
Thuny F, Beurtheret S, Mancini J, et al. The timing of surgery influences mortality and morbidity in adults with severe complicated infective endocarditis: A propensity analysis. Eur Heart $\mathrm{J}$ in adults with severe complicated infective endocarditis: A

2011;32(16):2027-2033. https://doi.org/10.1093/eurheartj/ehp089
4. Murdoch DR, Corey GR, Hoen B, et al. Clinical presentation, etiology, and outcome of infective Murdoch DR, Corey GR, Hoen B, et al. Clinical presentation, etiology, and outcome of infective
endocarditis in the 21st century. Arch Intern Med 2009;169(5):463-473. https://doi.org/10.1001/ archinternmed.2008.603

5. Slipczuk L, Codolosa JN, Davila CD, et al. Infective endocarditis epidemiology over five decades A systematic review. PLoS One 2013;8(12):e82665. https://doi.org/10.1371/journal.pone.0082665

6. Prendergast BD. The changing face of infective endocarditis. Heart 2006;92(7):879-885. https://doi. org/10.1136/hrt.2005.067256

Benito N, Miró JM, de Lazzari E, et al. Health care-associated native valve endocarditis: Importance of non-nosocomial acquisition. Ann Intern Med 2009;150(9):586-594. https://doi.org/10.7326/00034819-150-9-200905050-00004

8. Nadji G, Rusinaru D, Rémadi JP, Jeu A, Sorel C, Tribouilloy C. Heart failure in left-sided native valve infective endocarditis: Characteristics, prognosis, and results of surgical treatment. Eur J Heart Fail 2009;11(7):668-675. https://doi.org/10.1093/eurihf/hfp077

9. García-Cabrera E, Fernández-Hidalgo N, Almirante B, et al. Neurological complications of infective endocarditis. Circulation 2013;127(23):2272-2284. https://doi.org/10.1161/circulationaha.112.000813

10. DiNubile MJ, Calderwood SB, Steinhaus DM, Karchmer AW. Cardiac conduction abnormalities complicating native valve active infective endocarditis. Am J Cardiol 1986;58(13):1213-1217. https:// doi.org/10.1016/0002-9149(86)90384-X

11. Meine TJ, Nettles RE, Anderson DJ, et al. Cardiac conduction abnormalities in endocarditis defined by the Duke criteria. Am Heart J 2001;142(2):280-285. https://doi.org/10.1067/mhj.2001.116964

12. Meel R, Essop MR. Striking increase in the incidence of infective endocarditis associated with recreational drug abuse in urban South Africa. S Afr Med J 2018;108(7):585-589. https://doi. recreational drug abuse in urban So
org/10.7196/SAMJ.2018.v108i7.13007

13. Koegelenberg CFN, Doubell AF, Orth H, Reuter H. Infective endocarditis in the Western Cape Province of South Africa: A three-year prospective study. Q J Med 2003;96(3):217-225. https://doi. Province of South Africa: A
org/10.1093/qjmed/hcg028

14. Topan A, Carstina D, Slavcovici A, Rancea R, Capalneanu R, Lupse M. Assessment of the Duke criteria for the diagnosis of infective endocarditis after twenty-years: An analysis of 241 cases. Clujul Med for the diagnosis of infective endocarditis after twenty-y

15. Ohara T, Nakatani S, Kokubo Y, Yamamoto H. Clinical predictors of in-hospital death and early Ohara T, Nakatani S, Kokubo Y, Yamamoto H. Clinical predictors of in-hospital death and early
surgery for infective endocarditis : Results of CArdiac Disease REgistration (CADRE), a nation-wide surgery for infective endocarditis : Results of CArdiac Disease REgistration (CADRE), a nation-w
survey in Japan. Int J Cardiol 2013;167(6):2688-2694. https://doi.org/10.1016/jijcard.2012.06.117

survey in Japan. Int J Cardiol 2013;167(6):2688-2694. https://doi.org/10.1016/j.ijcard.2012.06.117
16. Chu VH, Cabell CH, Benjamin DK, et al. Early predictors of in-hospital death in infective endocarditis. Chu VH, Cabell CH, Benjamin DK, et al. Early predictors of in-hospital death in infective endof
Circulation 2004:109(14):1745-1749. https://doi.org/10.1161/01.cir.0000124719.61827.7f

17. Cahill TJ, Baddour LM, Habib G, et al. Challenges in infective endocarditis. J Am Coll Cardiol 2017;69(3):325-344. https://doi.org/10.1016/j.jacc.2016.10.066

18. Moreillon P, Que Y-A. Infective endocarditis. Lancet 2004;363(9403):139-149. https://doi.org/10.1016 s0140-6736(03)15266-X

9. Tornos P, Iung B, Permanyer-Miralda G, et al. Infective endocarditis in Europe: Lessons from the Euro heart survey. Heart 2005;91(5):571-575. https://doi.org/10.1136/hrt.2003.032128

20. Pant S, Patel NJ, Deshmukh A, et al. Trends in infective endocarditis incidence, microbiology, and valve replacement in the United States from 2000 to 2011. J Am Coll Cardiol 2015;65(19):2070-2076. https://doi.org/10.1016/j.jacc.2015.03.518

21. Dada S, Harker Burnhams N, Erasmus J, et al. South African Community Epidemiology Network of Drug Use (SACENDU): Monitoring alcohol, tobacco and other drug abuse treatment admission in South Africa. 2017. http://www.mrc.ac.za/sites/default/files/attachments/2017-05-22/ SACENDUPhase40.pdf (accessed 26 January 2019).

22. Njuguna B, Gardner A, Karwa R, Delahaye F. Infective endocarditis in low- and middle-income countries. Cardiol Clin 2017;35(1):153-163. https://doi.org/10.1016/..ccl.2016.08.011

23. Habib G, Lancellotti P, Antunes MJ, et al. 2015 ESC Guidelines for the management of infective endocarditis. Eur Heart J 2015;36(44):3075-3128. https://doi.org/10.1093/eurheartj/ehv319

24. Mirabel M, Rattanavong S, Frichitthavong K, et al. Infective endocarditis in the Lao PDR: Clinical characteristics and outcomes in a developing country. Int J Cardiol 2015;180:270-273. https//doi. org/10.1016/jijcard.2014.11.184

Accepted 5 February 2019. 